\title{
Thoracic Fluid Content Measurement: Diagnostic Value of Suspected Pulmonary Oedema in Acute Decompensate Heart Failure
}

\author{
(1) Ali Avcı ${ }^{1}$, (1) Mustafa Korkut ${ }^{2}$, (1) Ahmet Fırat Bektaș³, (1) Seçgin Söyüncü ${ }^{3}$ \\ ${ }^{1}$ Clinic of Emergency Medicine, Karaman State Hospital, Karaman, Turkey \\ ${ }^{2}$ Clinic of Emergency Medicine, University of Health Sciences Turkey, Antalya Training and Research Hospital, Antalya, Turkey \\ ${ }^{3}$ Department of Emergency Medicine, Akdeniz University, Antalya, Turkey
}

\begin{abstract}
Aim: One of the non-invasive methods of monitoring changes in cardiovascular haemodynamics is the measurement of transthoracic impedance (TTI). The most important features of this method are that it does not require catheterisation, it carries no risk of complications and it is both cost-effective and easily applicable.

The aim of this study was to determine the diagnostic value of thoracic fluid content (TFC), which is one the parameters obtained from TTI measurement in patients who present to the emergency department (ED) with dyspnoea and are suspected of pulmonary oedema (PO) due to acute decompensate heart failure (ADHF).

Materials and Methods: This single-centre, prospective, cohort clinical study was conducted on patients aged $>18$ years who presented to the tertiary care university ED with acute dyspnoea and were suspected of PO due to ADHF. The primary outcome measure was to determine the diagnostic value of TFC measurement in patients who presented to the ED with dyspnoea and were suspected of PO due to ADHF.

Results: A total of 113 patients were included in the study. The Brain Natriuretic Peptide (BNP) value was accepted as the gold standard in the diagnosis of PO due to ADHF. The sensitivity/specificity of PO associated with ADHF was 64.3/31.4\%, $14.3 / 88.4 \%$ and $60.5 / 83.7 \%$ for TFC, cardiac index $(\mathrm{Cl})$ and stroke index, respectively. Among these, only TFC was statistically significant in the diagnosis of $\mathrm{PO}(\mathrm{p}<0.001)$.
\end{abstract}

Conclusion: TFC measured by TTI in patients suspected of PO due to ADHF could be a better alternative for exclusion tests.

Keywords: Thoracic fluid content, transthoracic impedance, pulmonary oedema, emergency, stroke index, cardiac index

\section{Introduction}

Acute decompensated heart failure (ADHF) constitutes a significant amount of patients admitted to the emergency department (ED) with complaints of dyspnoea (1). Pulmonary oedema (PO) is one of the major causes of dyspnoea at the time of admission among these patients. Besides patient history and physical examination, other laboratory and imaging methods are included in the diagnosis of PO. In patients suspected of ADHF, Brain Natriuretic Peptide (BNP) and transthoracic echocardiography (TTE) are used most frequently as useful diagnostic bedside tests (2). The major disadvantage of BNP testing is that it cannot be obtained immediately. Lower and upper limit values should be used to obtain accurate sensitivity and specificity. Values below the lower limit provide excellent sensitivity and can be useful in excluding heart failure. Values above the upper limit present high specificity but the clinical presentation and examination findings of the patient should be taken into account in the diagnosis of heart failure (3). TTE is non-invasive and possible to be performed at the bedside, but it is an application-dependent diagnostic method. In addition, patients must be in a supine position for accurate evaluation. One of the non-invasive methods to monitor changes in cardiovascular haemodynamics is the measurement of transthoracic impedance cardiography (ICG). The most important features of this method is that it does not require catheterization, no risk of complications as well as being cost-effective and easily applicable $(4,5)$ ICG is a safe, non-invasive, and cheap (disposable tools used in the procedure cost about 7-8 euros or 8-9 US 
dollars (6). This ICG might be advantageous over BNP testing in patients admitted to the emergency department presenting with dyspnoea as its results can be obtained sooner ICG uses the difference between the electrical resistances of different tissues in the human body. For example, adipose tissue, bones, lungs and muscles are poor conductors of electricity while blood is a good conductor, and the blood flow and circulation result in a difference in chest impedance. Haemodynamic parameters are calculated from the impedance difference obtained after the measurement.

The inverse of the basal thoracic impedance relative to impact can give the amount of intrathoracic fluid. One of these parameters is the "amount of thoracic fluid" ICG is recorded by placing the electrodes in the longitudinal and lower thorax. Between the electrodes, a constant current of $400 \mu \mathrm{A}$ and a frequency of $40 \mathrm{kHz}$ is applied to the body and voltage is generated. Internal electrodes detect thoracic voltage changes. These voltage changes are used to calculate the impedance change. The detected static impedance indicates thoracic fluid volume, while the dynamic impedance is affected by aortic blood volume and velocity. Impedance is calculated according to Ohm's law. According to Ohm's Law; the current (I) of the electrical circuit is equal to the voltage drop (E) between the two ends of the circuit divided by the impedance (Z). From this impedance, hemodynamic parameters are calculated using mathematical methods. I=E/Z. Thoracic impedance is the electrical resistance of the thorax against high frequency low density current. This impedance is indirectly dependent on the thoracic fluid content. Recorded impedance difference is used for the measurement of hemodynamic parameters. ICG measurements can be obtained by a nurse or a technician in a few minutes without any necessary blood test (7). The following parameters can be calculated: Cardiac Index (CI), cardiac output (impedance cardiography CO), total peripheral vascular resistance, pulse volume and TFC. The presence of PO is also supported by TFC obtained via ICG. When the literature was reviewed, there were not found many studies showing the diagnostic value of ICG in the diagnosis of ADHF in patients admitted to the ED with dyspnoea.

The aim of this study was to determine the diagnostic value of the parameters obtained from ICG (especially TFC) in patients presented to the ED with dyspnoea, who were suspected of PO due to ADHF.

\section{Materials and Methods}

\section{Patient Selection}

This study was designed as a clinical prospective cohort study. Sample size was not calculated before the study. The patient population consisted of patients who presented to the ED of the tertiary university hospital with complaints of dyspnoea between 01.11.2017-10.02.2018. This study was approved by Akdeniz University Scientific Research Projects Coordination Unit (project number: TTU-2017-26434). Informed Consent was obtained from all of the patients. The study was in accordance with The Declaration of Helsinki. Patients admitted to the ED with dyspnoea, who were $\geq 18$ years old and pre-diagnosed with ADHF after clinical evaluation by an emergency physician. A total of 126 patients with complaints of dyspnoea were admitted during the period of the study. One hundred and thirteen patients were included in the study. The diagnosis of PO due to ADHF was verified according to the universally accepted techniques (2). We did not use TTE for diagnosis of ADHF. ADHF in this study were defined by using conventional techniques (an interview included age, dyspnoea and anamnesis; objective examination consisted of height, weight, respiration rate, pulmonary auscultation, oedemas, blood pressure, and heart rate, chest X-ray and BNP) and ICG. According to these sign and symptoms, non-cardiogenic and cardiogenic PO was defined. The following diagnostic values were used for the diagnosis of PO due to ADHF: BNP $>500 \mathrm{pq} /$ $\mathrm{mL}, \mathrm{TFC}>34.21 / \mathrm{kOhm}, \mathrm{Cl}>2.6 \mathrm{~L} / \mathrm{min} / \mathrm{m}^{2}$, Stroke index $(\mathrm{SI})>31.8$ $\mathrm{mL} / \mathrm{m}^{2}$. Each reference values are universal for the diagnosis of PE due to ADHF. The patients were categorized as four groups according to each parameter of ICG measurements. Group 1 as defined PE due to ADHF using by BNP, group 2 as defined PE due to ADHF using by TFC group 3 as defined PE due to ADHF using by $\mathrm{Cl}$ and group 4 as defined PE due to ADHF using by SI. BNP value was accepted as the gold standard in the diagnosis of PE due to ADHF. To show the diagnostic efficacy of ICG parameters, the other groups were compared based on the diagnosis of $\mathrm{PE}$ due to ADHF using BNP value. According to the accepted guidelines for ICG examination patients were excluded from the study if they had any of excluding criteria: excessive physical activity during registration $(n=1)$, severe pleural effusion $(n=2)$, known of severe aortic or mitral valve failure $(n=2)$, excessive low weight $<25 \mathrm{~kg}$ $(n=1)$, excessive high weight $>220 \mathrm{~kg}(n=1)$, short length $<120$ $\mathrm{cm}(\mathrm{n}=1)$, heart rate $>250$ beat/minute $(n=1)$, asthma $(n=1)$, Chronic obstructive pulmonary disease $(n=1)$, pneumonia $(n=1)$, pneumothorax $(n=1)$. These conditions cause to the effect of the poor quality of the ICG signal and the errors in the calculated parameters. Patients flow chart are shown in Figure 1.

\section{Study Protocol}

In this study, we used a (Task Force Monitor) transthoracic ICG monitor with 8 electrodes produced by the Austria company "CNSystems, Medizintechnik GmbH" The ICG measurement device was installed in the monitored ED. Emergency physicians received detailed training on how to monitor patients, where to 


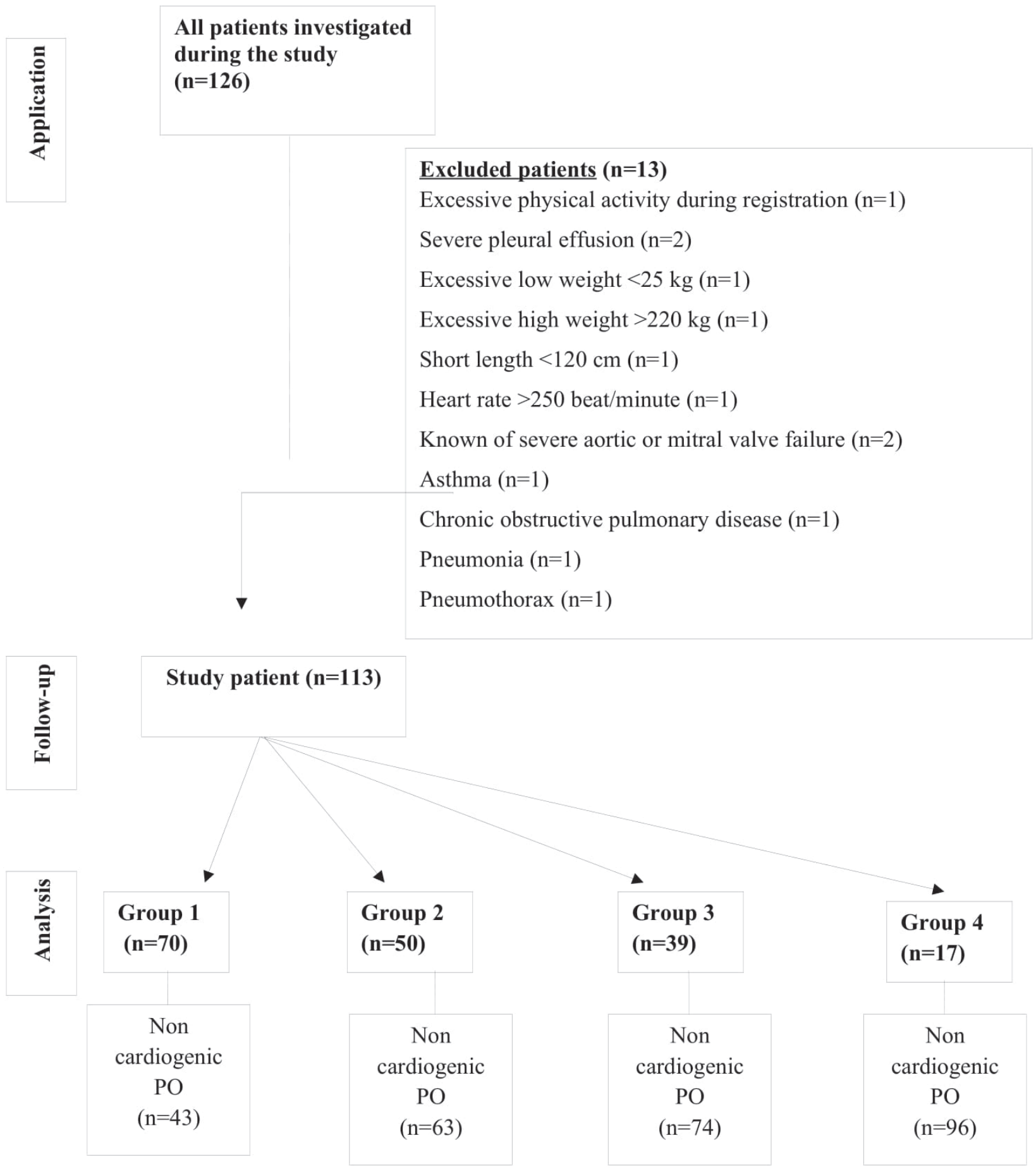

Figure 1. Patient flow chart

place the electrodes and how to record them. ICG was performed after a 10-min rest, the patient being in the lying position, with the vertex of the head elevated by $30^{\circ}$. The monitor measured and recorded continuous cardiovascular parameters for one minute after recording the gender, age, height and weight. The sensors measure the baseline impedance of the thorax. Impedance changes with each beat of the heart due to changes in the volume and velocity in the aorta. This produces a change in the electrical resistance (impedance) of the thorax to electrical alternating current. The changes in impedance can be used to measure or calculate hemodynamic parameters. In Figures 2 and 3, the electrodes used in the study are shown on the patient. The values of hemodynamic parameters such as TFC, $\mathrm{CO}, \mathrm{Cl}$, heart rate and $\mathrm{SI}$ were obtained by ICG and recorded in the patient follow-up form. The main parameters of ICG are presented in Table 1. 


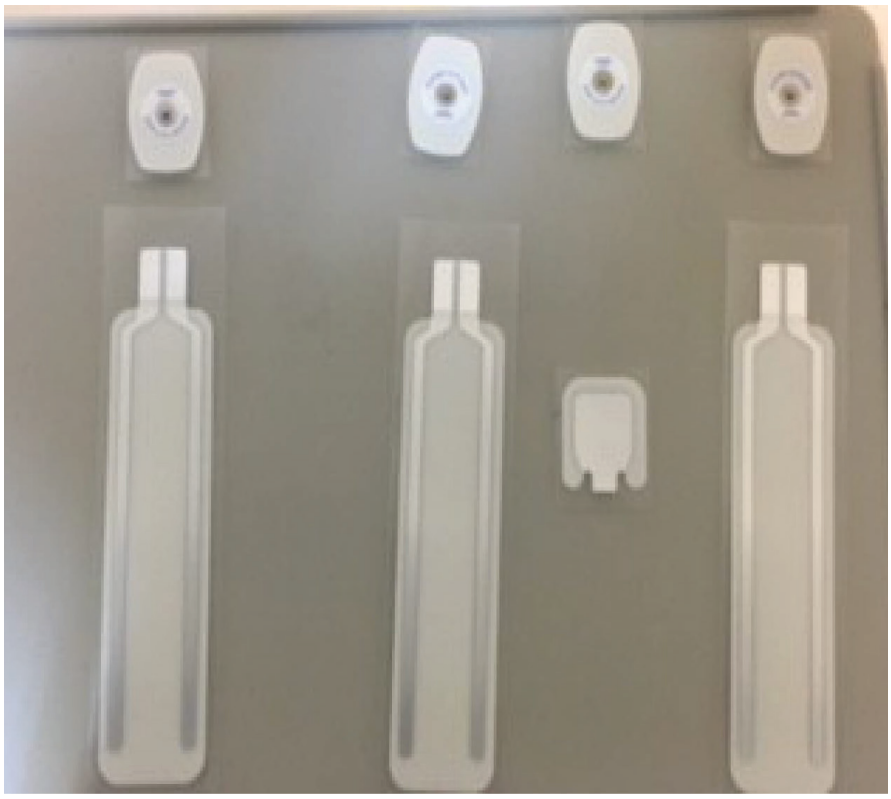

Figure 2. Electrodes used in the study (four electrodes on the upper that monitorization for patient, at lower one of them for static grounding and other three electrodes for impedance measure)

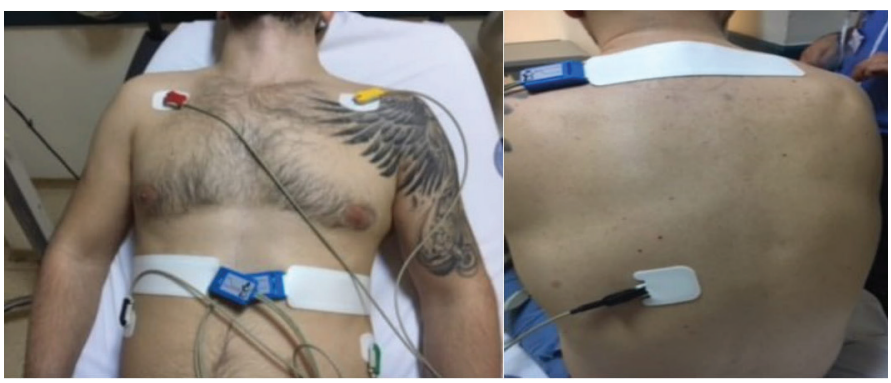

Figure 3. Electrodes were placed bilaterally at the base of the neck and on the thorax at the xiphoid level in the midaxillary line on the patient: the patient being in the lying position, with the vertex of the head elevated by $30^{\circ}$

\begin{tabular}{|l|l|l|}
\hline \multicolumn{2}{|l|}{ Table 1. The main parameters of impedance cardiography } \\
\hline ICG parameters & Normal value & $\begin{array}{l}\text { + Standardised values } \\
\text { used to diagnosis of } \\
\text { ADHF, mean } \pm \text { SD }\end{array}$ \\
\hline \multirow{2}{*}{ TFC } & Male: $30-501 / \mathrm{kOhm}$ & $32.5(31.0-37.7)$ \\
\cline { 2 - 3 } & Female:21-37 1/kOhm & \\
\hline SI & $30-65 \mathrm{~mL} / \mathrm{m}^{2}$ & $26.2(24.7-27.0)$ \\
\hline CI & $2.5-4.7 \mathrm{l} / \mathrm{min}^{2} / \mathrm{m}^{2}$ & $2.4(2.3-2.5)$ \\
\hline $\begin{array}{l}\text { ICG: Impedance cardiography, ADHF: Acute decompensated heart failure, TFC: } \\
\text { Thoracic Fluid Content, SI: Stroke index, Cl: Cardiac index, SD: Standard deviation } \\
\text { Standardised values used to diagnosis of ADHF: TFC }>34.21 / \mathrm{kOhm}, \mathrm{Cl}>2.6 \mathrm{~L} / \mathrm{min} / \\
\mathrm{m}^{2}, \mathrm{SI}>31 .{ }^{8 \mathrm{~m}} \mathrm{~L} / \mathrm{m}^{2}\end{array}$ \\
\hline
\end{tabular}

\section{Data Collection}

A data collection form was created to collect sociodemographic characteristics, clinical and laboratory findings, radiological results of the patients. Two trained, licensed emergency physicians independently reviewed all medical notes, including ED visit forms, hospital admission notes, and hospital discharge notes. The primary diagnosis of PO due to ADHF was identified by the emergency physician based on the results of conventional techniques and ICG parameters. To eliminate the possibility of bias, the reviewing physicians were blinded to results of BNP, ICG parameters.

\section{Statistical Analysis}

The statistical analysis of data was performed using SPSS 18.0 (SPSS, Inc., Chicago, IL) software. Percentage and number values were given for the variables grouped in the descriptive analyses while the mean values and 95\% confidence interval (Cl) were given for the other variables. The Shapiro-Wilks test was used for normal distribution prior to the comparison of the mean values. The Mann-Whitney U test was used to compare the averages in the non-normal distributed groups, and t-test was used to compare the averages of normally distributed variables in the dependent groups. Chi-square test was used for categorical variables. The Receiving operator curve (ROC) analysis was performed in the statistically significant group and the area under curve (AUC) was calculated to determine the predictive values. For all variables, $p<0.05$ was considered statistically significant.

\section{Results}

Out of a total of 126 patients who presented with dyspnoea, 113 patients were included in the study after the application of the exclusion criteria (Figure 1). The demographic status of patients is presented in Table 2. In our study, 74 (65.5\%) of the patients were male and 39 (34.5\%) were female. The mean age was 72.0 (70.0$75.0 \% 95 \% \mathrm{Cl}$ ), and the mean body mass index (BMI) was found 27 (27.2-29.4 95\% Cl) kg/m². The primary follow-up parameter of this study was to determine the diagnostic value of TFC value, which is one of the ICG parameters, for the diagnosis of PO due to ADHF. As a result of the tests carried out for the diagnosis of heart failure, the mean of the variables for TFC, SI, CI and BNP were determined as $32.51 / \mathrm{kOhm}[31.0-37.7 \%$ 95\% confidence interval (Cl], $26.2 \mathrm{~mL} / \mathrm{m}^{2}$ (24.7-27.0), 2,4 L/min/m² (2.3-2.5 95\% $\mathrm{Cl})$, and $655.0 \mathrm{pg} / \mathrm{dL}(556.1-814.495 \% \mathrm{Cl})$, respectively. There was found a statistically significant difference when the mean values of BNP and TFC were compared (Figure 4) $(p<0.001)$. PE due to ADHF was detected in $61.9 \%$ of the patients according to BNP value, $44.2 \%$ according to TFC, 34.8\% according to $\mathrm{Cl}$ and $15 \%$ according to SI. In addition, one of the patients presented with $\mathrm{MI}$ as a major cardiac adverse event (MI, cerebrovascular accident, arrhythmia, peripheral embolism), which occurred in the period of one-week follow-up. Other characteristics along with the findings of physical examination and chest X-ray are shown in Table 2. 
Based on BNP, the diagnostic sensitivity of PE associated with ADHF was $64.3 \%, 31.4 \%$, and $14.3 \%$ for TFC, CI, SI, respectively. Among these, only TFC was statistically significant $(p<0.001)$ (Table 3). ROC analysis was performed to distinguish the PE due to ADHF which was based on BNP by using the standardized values for TFC, SI and CI. TFC had the highest AUC value $(0.76, \mathrm{p}<0.001)$. The AUC was determined as 0.49-0.46 for $\mathrm{SI}$ and $\mathrm{CI}$, respectively. These findings indicate that only TFC is able to make a distinctive diagnosis for PE due to ADHF based on BNP (Table 4). ROC curves for TFC, SI and $\mathrm{Cl}$ and PE diagnosis due to ADHF based on BNP are shown in Figure 5. In our study, the optimal cut-off value of TFC for the diagnosis of PE due to ADHF was found 32.70. According to this cut-off value, the sensitivity was calculated as $70.0 \%$ and the specificity was $86.0 \%$ (Table 4). We examined the correlation between BMI and gender and TFC. The comparison of the mean

\begin{tabular}{|c|c|}
\hline \multicolumn{2}{|l|}{ Variable total $(n=113)$} \\
\hline Female/Male, $\mathbf{n}(\%)$ & $39(34.5) / 74(65.5)$ \\
\hline Age, mean \pm SD & $72(70.0-75.0)$ \\
\hline BMI, $\mathrm{kg} / \mathrm{m}^{2}$, mean $\pm \mathrm{SD}$ & $27.7(27.2-29.4)$ \\
\hline \multicolumn{2}{|l|}{ BMI, n (\%) } \\
\hline Normal & $35(31.0)$ \\
\hline Overweight & $39(34.5)$ \\
\hline Obese & $33(29.2)$ \\
\hline Morbid obese & $6(5.3)$ \\
\hline \multicolumn{2}{|l|}{ Pulmonary auscultation, n (\%) } \\
\hline Normal & $16(14.2)$ \\
\hline Crackle on the bilateral basal & $50(44.2)$ \\
\hline Crackle on the mid zone & $24(21.2)$ \\
\hline Crackle on the apex & $1(0.9)$ \\
\hline Other & $22(19.5)$ \\
\hline \multicolumn{2}{|l|}{ The sign of chest X-ray, n (\%) } \\
\hline Pulmonary oedema & $39(34.5)$ \\
\hline Inpatient from ED & $49(43.4)$ \\
\hline Discharge from ED & $62(54.9)$ \\
\hline MACE for during seven days & $1(0.9)$ \\
\hline
\end{tabular}

gender and BMI based on TFC revealed a statistically significant difference between the groups (Table 5).

The ROC analysis was used to evaluate the subgroups by BMI and gender separately for TFC based on the diagnosis of PO due to ADHF using BNP value. In normal weight, obese and morbid obese patients the values were determined as follows: the AUC

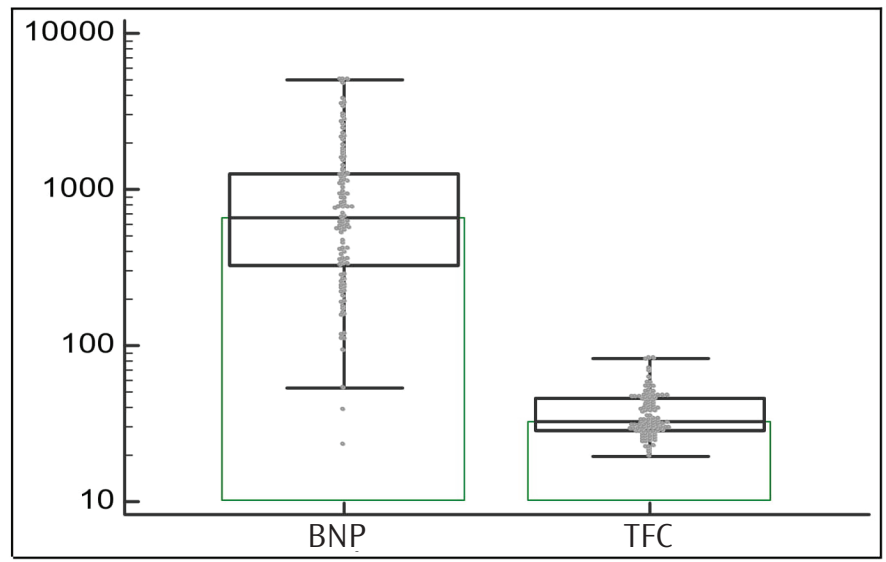

Figure 4. Comparison between the median values of BNP and TFC BNP: Brain natriuretic peptide, TFC: Thoracic fluid content

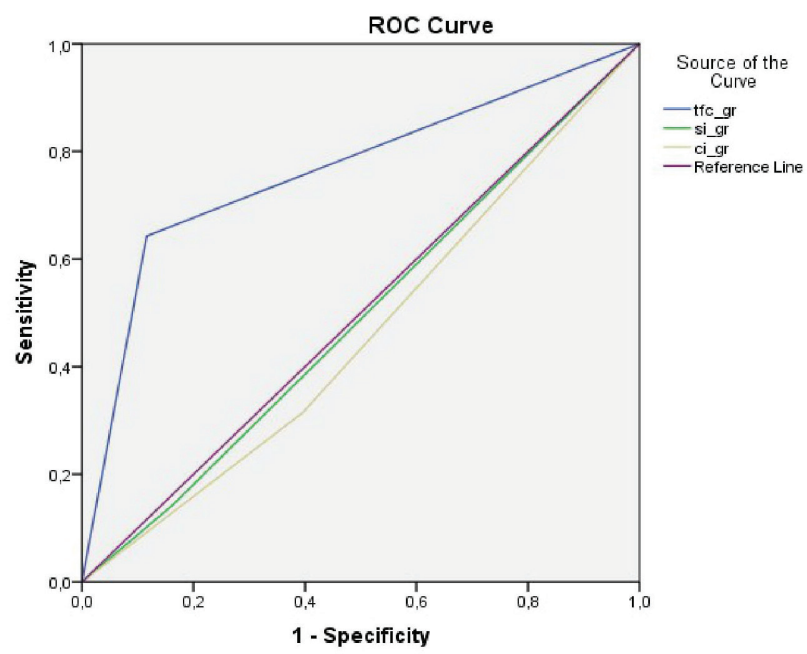

Figure 5 a. The TFC has a higher AUC [0.76 (0.674-0.853), $p<0.001]$ than SI and CI respectively AUC [0.49 (0.380-0.600)]

\#Standardized values used to TFC, SI and Cl

TFC: Thoracic fluid content, AUC: Area under curve, SI: Stroke index, $\mathrm{Cl}$ : Cardiac index

Table 3. Comparison of PO due to ADHF between the BNP and TFC, SI, CI

\begin{tabular}{|c|c|c|c|c|c|}
\hline Variable total $(n=113)$ & n (\%) & $\begin{array}{l}\text { PO due to ADHF using by } \\
\text { BNP (group 1), n (\%) }\end{array}$ & Sensitivity & Specificity & $\# p$ \\
\hline PO due to ADHF using by TFC (group 2) & $50(44.2)$ & $45(64.3)$ & 64.3 & 88.4 & $<0.001$ \\
\hline PO due to ADHF using by SI (group 4) & $17(15)$ & $10(14.3)$ & 14.3 & 83.7 & 0.774 \\
\hline
\end{tabular}


$(0.85,0.83)$, optimal cut-off values $(36.45,31.77)$ and sensitivityspecificity $(77-89,71-85)$, respectively. The ROC analysis was used to evaluate the subgroups by gender separately for TFC based on the diagnosis of PE due to ADHF using BNP value. In male and female patients, the values were determined as follows: the AUC $(0.84,0.81)$, optimal cut-off points $(32.7,31.8)$, sensitivityspecificity $(65-95,78-79)$ based on these values, respectively. (Figure 6)

\section{Discussion}

In this study, we investigated the diagnostic value of TFC, which is one of ICG parameters, in patients presented to the ED with dyspnoea and suspected of PO due to ADHF. BNP was determined as the gold standard for the diagnosis of PO due to ADHF. In addition to the high diagnostic value of TFC, it was concluded that it could also replace other exclusion tests. Besides, ICG has been proven to be a valid and accurate method in some cardiovascular diseases and emergency medical conditions, and

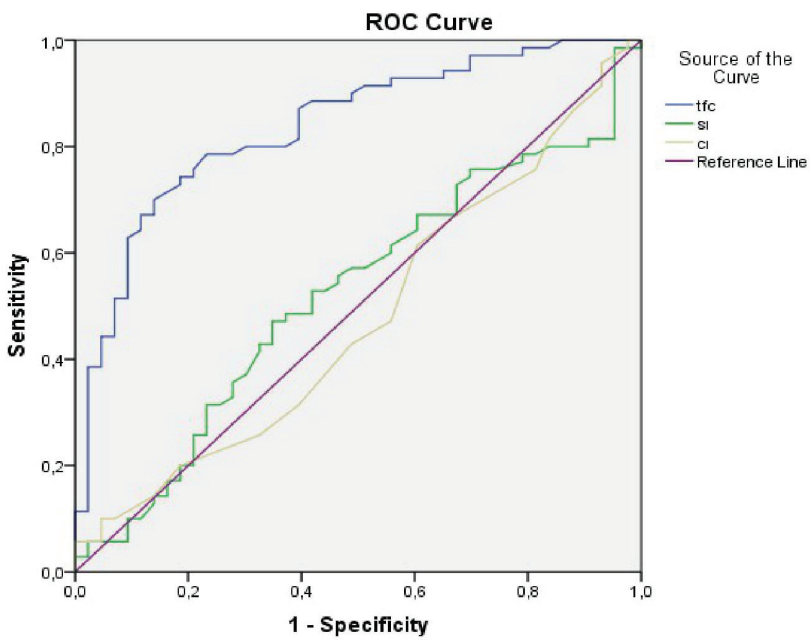

Figure $5 \mathbf{b}$ b. $>32.701 / \mathrm{kOhm}$ value used to TFC and AUC $[0.83$ $(0.762-0.912), p<0.001]$ was found

TFC: Thoracic fluid content, AUC: Area under curve

Table 4. ROC curve analysis of each parameters for PO due to ADHF using by BNP

\begin{tabular}{|l|l|l|l|l|}
\hline & Sensitivity & Specificity & AUROC & $\mathbf{p}$ \\
\hline TFC, \% & 64.3 & 88.4 & $0.76(0.674-0.853)$ & $<\mathbf{0 . 0 0 1}$ \\
\hline *TFC, \% & 70 & 86 & $0.83(0.762-0.912)$ & $<\mathbf{0 . 0 0 1}$ \\
\hline SI, \% & 14.3 & 83.7 & $0.49(0.380-0.600)$ & $\mathbf{0 . 8 5 9}$ \\
\hline CI, \% & 31.4 & 60.5 & $0.46(0.349-0.570)$ & 0.471 \\
\hline
\end{tabular}

AUROC: Area under the receiver operating characteristic curve, PO: Pulmonary oedema, ADHF: Acute decompensated heart failure, ROC: Receiver operating Characteristic curve

Standardised values used to diagnosis of ADHF: BNP $>500 \mathrm{pq} / \mathrm{mL}$, TFC $>34,21 /$ $\mathrm{kOhm}, \mathrm{Cl}>2.6 \mathrm{~L} / \mathrm{dk} / \mathrm{m}^{2}, \mathrm{SI}>31.8 \mathrm{~mL} / \mathrm{m}^{2}$

*Cut off for TFC $>3.701 / \mathrm{kOhm}$ in the study could be substituted for other invasive diagnostic methods by virtue of its non-invasive nature (8). The literature reveals that physical examination alone is not enough for the diagnosis of $\mathrm{PO}$ due to $\operatorname{ADHF}(9,10)$. Additional diagnostic methods are needed. In cardiac failure, the routine evaluation where a catheter is passed into the chambers of the heart carries a risk of serious complications. This can be facilitated by the application of ICG. Consistent with the literature, we also found $64.3 \%$ sensitivity and $88.4 \%$ specificity for TFC $\geq 34.21 / \mathrm{kOhm}$ when BNP value was determined as the gold standard for the diagnosis of PO due to ADHF. Malfatto et al. included 29 patients of a $72 \pm 4$ year age group with NHYA class of $3.5 \pm 0.9$ (New York Heart Association) and an ejection fraction of $28 \pm 6 \%$. They obtained the value of TFC by ICG, and measured pulmonary capillary wedge pressure
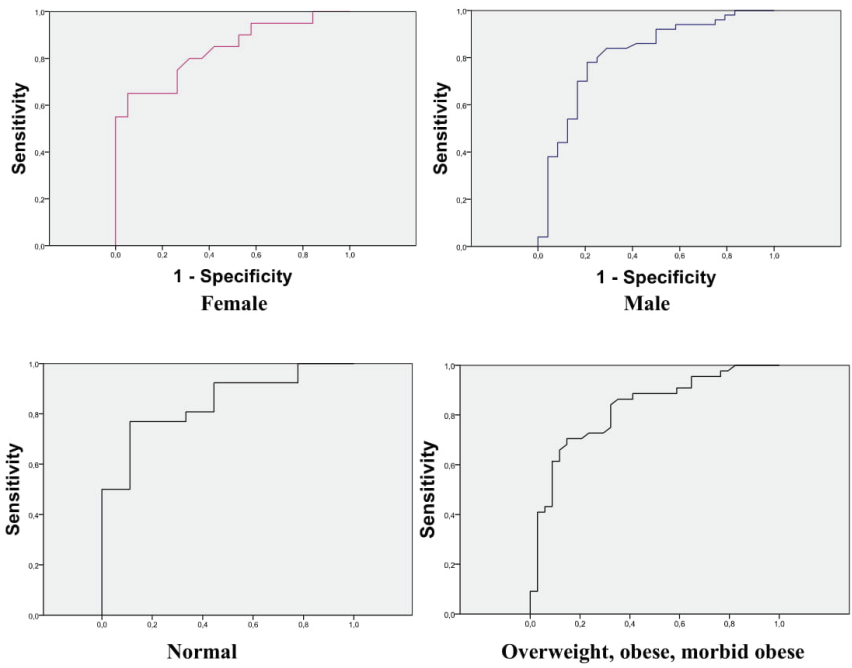

Figure 6. Receiver operation characteristic curve for PO due to ADHF using by TFC (Groups was estimated separately according to $\mathrm{BMI}$ and genders)

PO: Pulmonary oedema, ADHF: Acute decompensated heart failure, TFC: Thoracic fluid content, BMI: Body mass index

Table 5. Comparison between the BMI and gender according to TFC

\begin{tabular}{|c|c|c|c|c|c|c|}
\hline & $n$ & $\begin{array}{l}\text { Mean } \\
\text { rank }\end{array}$ & $\begin{array}{l}\text { Sum of } \\
\text { rank }\end{array}$ & $\mathrm{U}$ & Z & $\# p$ \\
\hline \multicolumn{7}{|l|}{ Gender } \\
\hline Female & 39 & 48.36 & 1886 & \multirow[t]{2}{*}{1106} & \multirow[t]{2}{*}{-2.035} & \multirow[t]{2}{*}{0.042} \\
\hline Male & 74 & 61.55 & 4555 & & & \\
\hline \multicolumn{7}{|l|}{ BMI } \\
\hline Normal & 35 & 70.83 & 2479 & \multirow[b]{2}{*}{881} & \multirow[b]{2}{*}{-3.006} & \multirow[b]{2}{*}{0.003} \\
\hline $\begin{array}{l}\text { Overweight, obese, } \\
\text { morbid obese }\end{array}$ & 78 & 50.79 & 3962 & & & \\
\hline
\end{tabular}

BMI: Body mass index, TFC: Thoracic Fluid content, U: u test statistic, Z: z test statistic

\#Mann-Whitney U test was used 
(PCWP) using cardiac catheterization. The value of TFC $\geq 351 / \mathrm{kOhm}$ obtained by ICG had high specificity (97\%) and sensitivity (86\%) in patients with $P C W P \geq 15 \mathrm{mmHg}$ using cardiac catheterization. The reproducible and non-invasive nature of ICG and the fact that the accuracy of TFC was also proved in our study indicate that ICG can be successfully used in the diagnosis and follow-up of the treatment. It is thought that ICG can substitute for BNP testing in patients who were presented with dyspnoea and suspected of $\mathrm{PO}$ due to ADHF as it has a similar diagnostic value. Because ICG measurement also has advantages over BNP. In many studies positive results have been obtained between BNP and ICG in patients with ADHF. A study on 331 patients showed that BNP and STR (the marker of contractly) on ICG were good prognostic factors when evaluating heart failure flare-up $(11,12)$. Another study involving 524 patients showed that ICG, STR, and BNP could be indicators of early heart failure flare-up, and the relative risk of heart failure flare up increased by 12.5 times $(p<0.001)$ at BNP $>100 \mathrm{pg} / \mathrm{mL}$ and STR $>0.45$ (11). In the presence of PO, ICG can be used in the distinctive diagnosis of dyspnoea in the $\operatorname{ED}(13,14)$. A study including 120 heart failure patients showed that TFC $>35$ $1 / \mathrm{kOmega}$ measured by ICG together with BNP $>350 \mathrm{pg} / \mathrm{mL}$ had $95 \%$ sensitivity and $96 \%$ specificity when diagnosing diastolic heart failure (15). Although BNP monitoring can be performed at the bedside, a laboratory is also required. Time is crucial for these patients requiring urgent treatment. A study reveals that it takes more than one hour from the beginning of BNP testing until the physician obtains the results and proceed with the assessment for diagnosis and treatment accordingly (16). ICG can be performed in less time and replicated if necessary. In our study, the optimal cut-off value for the diagnosis of PO due to ADHF was found 32.70. Replication of the statistical methods based on this cut-off value revealed $70.0 \%$ sensitivity and $86.0 \%$ specificity. We used the value of 34.20 as a standardized cut-off point for some findings in our study, which was previously found as an optimal cut-off value in a study (17). It could be concluded that the cut-off value for TFC could be standardized and used in the diagnosis of PO due to ADHF as these values were similar to each other. The mean age of the study was 72.0 years. In other studies, the mean age was over 65 years, which is similar to our study showed that TFC might vary based on anthropometric measurements (height, weight) and gender (17-19). Similarly, we found a statistically significant difference between the groups for both variables when we compared the mean values of TFC based on gender and BMI in our study. The optimal cut-off values of TFC varied according to gender and BMI when evaluated by ROC analysis. This suggests that TFC may have a higher diagnostic value when different cut-off values are accepted for TFC according to gender and BMI. However, more extensive studies are required in this regard.

\section{Study Limitations}

There were several limitations in our study. Firstly, it was a singlecentred study. Secondly, the study sample was small. We didn't use TTE for diagnosis of ADHF therefore BNP value was accepted as the gold standard in the diagnosis of PO due to ADHF. Patients with arrhythmias were included in the study. This condition may have affected our results. We did not follow up non-cardiogenic PE and the outcomes of hospitalised patients. Other study limitations could be the adaptation period to the ICG device by emergency physician as there had not been any similar study or application in the ED prior to our study, and the difficulty faced by symptomatic patients to lie in the supine position. This study will be a step forward in prospective studies with larger patient populations.

\section{Conclusion}

Patients with PO due to ADHF are referred to the ED in an agitated and severe condition. Therefore, our study has revealed the importance of the use of ICG device in the ED as it is fast, noninvasive and can be performed at the bedside. In conclusion, this study showed that TFC obtained by ICG device has a high diagnostic value for PO and it can also be used as an exclusion method.

\section{Ethics}

Ethics Committee Approval: This study was supported by Akdeniz University Scientific Research Projects Coordination Unit (project number: TTU-2017-26434).

Informed Consent: Informed consent was obtained from all of the patients.

Peer-review: Externally and internally peer-reviewed.

\section{Authorship Contributions}

Concept: A.A., A.F.B., S.S., Design: A.A., A.F.B., Data Collection or Processing: A.A., A.F.B., Analysis or Interpretation: M.K., A.F.B., Literature Search: A.A., M.K., A.F.B., S.S., Writing: A.A., M.K.

Conflict of Interest: The authors declare that they have no conflict of interest.

Financial Disclosure: There are no financial supports.

\section{References}

1. Pang PS, Collins SP, Sauser K, Andrei A-C, Storrow AB, Hollander JE, et al. Assessment of Dyspnea Early in Acute Heart Failure: Patient Characteristics and Response Differences Between Likert and Visual Analog Scales. Hiestand B, editor. Acad Emerg Med. 2014;21:659-66.

2. Ponikowski P, Voors AA, Anker SD, Bueno H, Cleland JGF, Coats AJS, et al. 2016 ESC Guidelines for the diagnosis and treatment of acute and chronic heart failure: The Task Force for the diagnosis and treatment of acute and chronic 
heart failure of the European Society of Cardiology (ESC) Developed with the special contribution o. Eur Heart J. 2016;37:2129-200.

3. Collins SP, Storrow AB. Acute Heart Failure. In: Tintinalli Stapczynski JS, Ma OJ, Yealy DM, Meckler GD CD. Tintinalli's Emergency Medicine: A Comprehensive Study Guide, 8e | AccessMedicine | McGraw-Hill Medical.https://accessemergencymedicine.mhmedical.com/content. aspx?sectionid $=109428688 \&$ bookid $=1658$

4. Becker K. Resolved: A pulmonary artery catheter should be used in the management of the critically ill patient. Con. J Cardiothorac Vasc Anesth. 1998;12(2 Suppl 1):13-6

5. Hendrickson K. Cost-effectiveness of noninvasive hemodynamic monitoring. AACN Clin Issues. 1999;10:419-24.

6. Sadauskas S, Naudžiūnas A, Unikauskas A, Mašanauskienė E, Bakšytė G, Macas A. Applicability of Impedance Cardiography During Heart Failure Flare-Ups. Med Sci Monit. 2016;;22:3614-22.

7. Summers RL, Shoemaker WC, Peacock WF, Ander DS, Coleman TG. Bench to bedside: electrophysiologic and clinical principles of noninvasive hemodynamic monitoring using impedance cardiography. Acad Emerg Med. 2003;10:669-80.

8. Pranulis MF. Impedance cardiography (ICG) noninvasive hemodynamic monitoring provides an opportunity to deliver cost effective, quality care for patients with cardiovascular disorders. J Cardiovasc Manag. 2000;11:13-7.

9. Peacock WF. The B-type natriuretic peptide assay: a rapid test for heart failure. Cleve Clin J Med. 2002;69:243-51.

10. Shah MR, Stinnett SS, McNulty SE, Gheorghiade M, Zannad F, Uretsky B, et al. Hemodynamics as surrogate end points for survival in advanced heart failure: An analysis from FIRST. Am Heart J. 2001;141:908-14.

11. Castellanos LR, Bhalla V, Isakson S, Daniels LB, Bhalla MA, Lin JP, et al. B-Type Natriuretic Peptide and Impedance Cardiography at the Time of Routine Echocardiography Predict Subsequent Heart Failure Events. J Card Fail. 2009;15:41-7.
12. Bhalla V, Isakson S, Bhalla M, Lin J, Clopton P, Gardetto N, et al. Diagnostic ability of B-type natriuretic peptide and impedance cardiography: Testing to identify left ventricular dysfunction in hypertensive patients. Am J Hypertens. 2005;18:73-81.

13. Peacock WF, Albert NM, Kies P, White RD, Emerman CL. Bioimpedance monitoring: better than chest x-ray for predicting abnormal pulmonary fluid? Congest Heart Fail. 2000;6:86-9.

14. Newman RB, Pierre H, Scardo J. Thoracic-fluid conductivity in peripartum women with pulmonary edema. Obstet Gynecol. 1999;94:48-51.

15. Malfatto G, Branzi G, Giglio A, Villani A, Facchini C, Ciambellotti F, et al. Transthoracic bioimpedance and brain natriuretic peptide levels accurately indicate additional diastolic dysfunction in patients with chronic advanced systolic heart failure. Eur J Heart Fail. 2010;12:928-35.

16. Peacock WF. Vein-to-brain Time: An Emergency Department Quality of Care Marker for non-ST-segment Elevation Acute Coronary Syndromes. Acad Emerg Med. 2004;11:569-70.

17. Peacock WF, Summers RL, Vogel J, Emerman CE. Impact of impedance cardiography on diagnosis and therapy of emergent dyspnea: The EDIMPACT trial. Acad Emerg Med. 2006;13:365-71.

18. Milzman D, Napoli A, Hogan C, Zlidenny A, Janchar T. Thoracic impedance vs chest radiograph to diagnose acute pulmonary edema in the ED. Am J Emerg Med. 2009;27:770-5.

19. Sanidas EA, Grammatikopoulos K, Anastasiadis G, Papadopoulos D, Daskalaki M, Votteas V. Thoracic fluid content and impedance cardiography: a novel and promising noninvasive method for assessing the hemodynamic effects of diuretics in hypertensive patients. Hellenic J Cardiol. 2009;50:465-71. 